Technical Note

\title{
Simplified longitudinal seismic response of tunnels linings subjected to surface waves
}

\author{
A.L. Sánchez-Merino, J. Fernández-Sáez, C. Navarro* \\ Department of Continuum Mechanics and Structural Analysis, Escuela Politécnica Superior, Universidad Carlos III de Madrid, Avda. Universidad 30, 28911-Leganés, Madrid, Spain
}

Keywords:

Tunnel seismic response

Soil-structure interaction

Surface waves

Tunnel longitudinal analysis

Timoshenko beam

\begin{abstract}
A B S T R A C T
This paper presents an engineering approach for analysing the longitudinal behaviour of tunnels subjected to earthquakes. The tunnel is modelled as a Timoshenko beam connected to the far soil by means of continuous elastic support (Winkler model). Seismic free field inputs, such as those caused by surface waves travelling parallel to the tunnel axis, were imposed at the base of the springs of the Winkler model, generating bending moments and shear forces on the cross sections of the tunnel. Closed form expressions of the tunnel displacements, shear forces, and bending moments were determined at any tunnel section in terms of the seismic excitation, tunnel geometry and material properties, and subgrade reaction modulus of the soil. A dimensional analysis was carried out to ascertain directly the maximum tunnel displacement, bending moment, and shear force.
\end{abstract}

\section{Introduction}

Seismic tunnel soil interaction is often minor because of the high damping in buried structures. One preliminary analysis of the seismic longitudinal response of tunnels may be performed while ignoring the physical presence of the tunnel and assuming that it undergoes exactly the same strains as the surrounding ground in free field conditions (absence of structures) for the different wave types that appear in a seismic event. However, this analysis method may overestimate or underestimate struc ture deformations, depending on the rigidity of the structure relative to the ground, and some field data indicate that stiff tunnels in soft soils rarely undergo strains equal to those of the soil [1].

Seismic input for shallow tunnel analysis at sites far from the seismic source is caused mainly by surface seismic waves (Rayleigh and Love waves) [2]. Although surface waves propagate in a random direction relative to the tunnel axis, the most penalising hypothesis, in relation to tunnel curvature, is the one in which surface waves travel parallel to tunnel axis [3].

One engineering alternative for complete tunnel seismic analysis is to carry out $3 \mathrm{D}$ full numerical analysis of the problem $[4,5]$. Alternatively, simplified models for seismic longitudinal lined tunnel analysis have been widely used. Constantopoulos

\footnotetext{
* Corresponding author. Tel.: +34916249491; fax: +34916249973.

E-mail address: navarro@ing.uc3m.es (C. Navarro).
}

et al. [6] developed a simple methodology in which the tunnel of finite length was modelled as a Winkler type beam, and soil free field motion caused by surface waves was imposed at the base of the springs that represented the tunnel soil interaction. These researchers solved the problem using the finite differences method. Using this methodology, Navarro and Samartín [7] obtained closed form expressions for the internal forces and bending moments in the tunnel. The most important feature of both papers is that seismic input was introduced at the base of springs approaching the peak part of the accelerogram by means of a parabola, and thus no assumptions about frequencies needed to be considered. St. John and Zahrah [8] also developed a Winkler type model to analyse long tunnels subjected to harmonic seismic waves, which gave closed form expression for the maxima axial and shear forces as well as bending moments acting on a tunnel section, and also the values of the spring constant to be used in the model, which were a function of the wave length, tunnel diameter, and elastic soil constants. In these last three references, the tunnel was assumed to behave as a Euler Bernoulli beam and thus shear deformation effects on tunnel structural response were not accounted for. Karadeniz [9] presented an interface $3 \mathrm{D}$ element for the linear dynamic analysis of buried or pile structures, under a seismic ground motion represented by Rayleigh waves, and assuming a viscoe lastic soil behaviour. In his formulation of the problem, he took into account the effects of shear deformation and a constant axial force. Also, he established the stiffness matrix and the load vector in the interface element, the latter being a function of the ground deformation, which leads to a matrix formulation of the problem. 
In this paper, a simplified longitudinal seismic analysis of a segmented tunnel is given. Because inertial response of tunnels is small due to the high radiation damping that appear in these kinds of problems, the analysis was carried out statically, greatly simplifying the problem solution. Closed form expressions for the tunnel displacements, shear forces and bending moments at any tunnel section (in terms of the seismic excitation), tunnel geometry, and material properties were determined and the results are shown in a dimensionless form, which allows the engineer to deduce the values of such magnitudes very easily. The tunnel is modelled as a finite Timoshenko beam to account properly for the shear deformation effects on the structural response of concrete lined tunnels, built in different phases and thus having construction joints, in which these effects become relevant because the diameter length ratios are relatively small. Additionally, the seismic accelerogram input is approximated by a parabola, which eliminates the need to treat the problem by Fourier analysis of different harmonic surface waves propagating along the tunnel axis. Because soil tunnel interaction changes the structural tunnel response regarding that determined when free field soil seismic strains are directly imposed to the tunnel, the problem is analysed taking into account this phenomenon in an approximate way.

\section{Statement and solution of the problem}

When a Rayleigh surface wave (polarised in a vertical plane) or a Love wave (polarised in a horizontal plane) travels parallel to the tunnel axis, the latter undergoes deformations. To determine the bending moment and shear force acting on the tunnel cross section, let us consider a tunnel of length $L$ modelled as a Timoshenko beam and connected to the far soil by a Winkler type model. The subgrade stiffness may be represented by $k$ per unit tunnel length and the far soil is subjected to a known displacement $w_{\mathrm{s}}(x)$, where the $x$ axis has the direction of the axis beam with its origin at the tunnel mid span section. As a consequence of soil movements, the tunnel will undergo the displacements $w(x)$.

To simplify the calculations, we assume that the part of the site accelerogram corresponding to the peak acceleration $a_{\text {max }}$ may be approximated by a parabolic curve as

$\frac{\mathrm{d}^{2} w_{s}(x)}{\mathrm{d} t^{2}}=a_{\max }\left[1 \quad\left(\frac{t}{\Delta T / 2}\right)^{2}\right]$

where the time scale now refers to the instant at which peak acceleration occurs at the tunnel mid span section and $\Delta T$ is the minimum value of $\Delta T_{1}$ and $\Delta T_{2}$ that are given, respectively, by $\Delta T_{1}=L / V$ and $\Delta T_{2}=T / 2$, with $V$ being the seismic wave velocity and $T$ the predominant earthquake period, as suggested by Constantopoulos et al. [6]. Because of surface waves show a dispersive character in layered soil systems, their low frequency wave velocities, such as the ones predominant in earthquakes $(15 \mathrm{~Hz})$, travel at speeds quite similar to those of the deeper layers that are, in general, much stiffer. This fact implies that expected surface wave lengths are usually of several hundred metres, whereas typical tunnel segment lengths are only a few metres long, and thus $\Delta T_{1}$ is usually much lower than $\Delta T_{2}$

If we denote $x=V \times t$ as the abscissa of a generic tunnel section from its mid span section, the peak zone of the accelerogram may be expressed as

$\frac{\mathrm{d}^{2} w_{\mathrm{s}}(x)}{\mathrm{d} x^{2}}=\frac{a_{\max }}{V^{2}}\left[1 \quad\left(\frac{2 x}{L}\right)^{2}\right]$
After integration twice, the seismic soil displacement may be calculated, resulting in

$w_{\mathrm{s}}(x)=\frac{a_{\max }}{V^{2}}\left[\begin{array}{lll}\frac{x^{2}}{2} & \frac{1}{L^{2}} \frac{x^{4}}{3} & \frac{5}{48} L^{2}\end{array}\right]$

Assuming that the tunnel behaves as a Timoshenko beam (neglecting the tunnel inertial forces) the deformed tunnel slope, $\mathrm{d} w(x) / \mathrm{d} x$, depends not only on the rotation of the tunnel cross section $\varphi_{y}(x)$ but also on the shear strain $\gamma_{x z}(x)$, so that magnitude may be expressed as

$\frac{\mathrm{d} w(x)}{\mathrm{d} x}=\varphi_{y}(x)+\gamma_{x z}(x)$

The bending moment $M$ and the shear force $Q$ at any tunnel section are given by

$M=E I \frac{\mathrm{d} \varphi_{y}(x)}{\mathrm{d} x}, \quad Q=A_{\mathrm{c}} \gamma_{x z} G=A_{\mathrm{c}} G\left(\frac{\mathrm{d} w}{\mathrm{~d} x}+\varphi_{y}(x)\right)$

where $E$ and $G$ are, respectively, the Young and Shear moduli of the beam material, $I$ is the inertia moment of the section regarding the neutral axis, and $A_{\mathrm{c}}$ the effective cross sectional area of the beam, related to the cross section area, $A$, by $A c=k_{\mathrm{ef}} A$, where $k_{\mathrm{ef}}$ the cross section shear coefficient (see, for instance, [10]).

The tunnel slide equilibrium conditions give two differential equations:

$E I \frac{\mathrm{d}^{2} \varphi_{y}(x)}{\mathrm{d} x^{2}} \quad A_{c} G\left(\frac{\mathrm{d} w(x)}{\mathrm{d} x}+\varphi_{y}(x)\right)=0$,

$A_{c} G\left(\frac{\mathrm{d}^{2} w}{\mathrm{~d} x^{2}}+\frac{\mathrm{d} \varphi_{y}(x)}{\mathrm{d} x}\right)=p(x)$

From these two equations, the following differential equation, in terms of the variable $\varphi_{y}(x)$, may be stated:

$\frac{\mathrm{d}^{4} \varphi_{y}(x)}{\mathrm{d} x^{4}} \quad \frac{k}{A_{\mathrm{c}} G} \frac{\mathrm{d}^{2} \varphi_{y}(x)}{\mathrm{d} x^{2}}+\frac{k}{E I} \varphi_{y}(x)=\frac{k}{E I} \frac{\mathrm{d} w_{\mathrm{s}}(x)}{\mathrm{d} x}$

This equation should fulfil four boundary conditions. Because of the symmetry of the problem, $\varphi_{y}(x)$ should be an odd function in $x$, becoming zero at $x=0$. On the other hand, because the bending moment and shear forces are zero at both tunnel edges sections, the first and second derivatives of $\varphi_{y}(x)$ should also become zero at $x= \pm L / 2$.

Solving Eq. (9) and imposing the boundary conditions, the bending moment and shear force at any section in the tunnel may be written as

$M=E I \frac{\mathrm{d} \varphi_{y}(x)}{\mathrm{d} x}=E I[2 C[\beta \sin \beta x \sinh \alpha x+\alpha \cos \beta x \cosh \alpha x]$

$\left.+2 D[\beta \cos \beta x \cosh \alpha x+\alpha \sin \beta x \sinh \alpha x]+p+3 r x^{2}\right]$

$$
\begin{aligned}
Q=\frac{\mathrm{d} M}{\mathrm{~d} x}= & E I\left[2 C\left[\begin{array}{lll}
\left(\alpha^{2}\right. & \left.\beta^{2}\right) \cos \beta x \sinh \alpha x & 2 \alpha \beta \sin \beta x \cosh \alpha x
\end{array}\right]\right. \\
& +2 D\left[\begin{array}{ll}
\left(\alpha^{2}\right. & \left.\left.\beta^{2}\right) \sin \beta x \cosh \alpha x+2 \alpha \beta \cos \beta x \sinh \alpha x\right]+6 r x
\end{array}\right]
\end{aligned}
$$

in which the new parameters $\alpha$ and $\beta$ are

$\alpha=\sqrt{\sqrt{\frac{k}{4 E I}}+\frac{k}{4 A_{\mathrm{c}} G}}$ and $\beta=\sqrt{\sqrt{\frac{k}{4 E I}}} \frac{k}{4 A_{\mathrm{c}} G}$

for values of $k$ that verifying $k<\left(4 A_{c}^{2} G^{2}\right) / E I$. In most practical cases, this last condition is fulfilled because the tunnel stiffness is greater than that of the soil, as suggested by Yin [11]. Although the value of the parameter $k$ depends on the soil layered system stiffness and tunnel geometry, St. John and Zahrah [8] proposed an expression of such a parameter. 
The rest of the parameters appearing in Eqs. (10) and (11) are summarised in Appendix A.

Tunnel displacements could be calculated from this differential equation:

$\frac{\mathrm{d}^{4} w(x)}{\mathrm{d} x^{4}} \quad \frac{k}{A_{\mathrm{c}} G} \frac{\mathrm{d}^{2} w(x)}{\mathrm{d} x^{2}}+\frac{k}{E I} w(x)=\frac{k}{E I} w_{\mathrm{s}}(x) \quad \frac{k}{A_{\mathrm{c}} G} \frac{\mathrm{d}^{2} w_{\mathrm{s}}(x)}{\mathrm{d} x^{2}}$

After solving this last equation, considering the corresponding boundary conditions cited for $\varphi_{y}(x)$, tunnel displacement at any section is

$w(x)=2 C_{\mathrm{w}} \cos \beta x \cosh \alpha x+2 D_{\mathrm{w}} \sin \beta x \sinh \alpha x$

$$
+p_{\mathrm{w}} x^{4}+q_{\mathrm{w}} x^{2}+r_{\mathrm{w}}
$$

The new coefficients that appear in this last equation are given in Appendix B.

\section{Dimensional analysis}

After the analytical solution of the problem, the results may be presented dimensionless. The maximal tunnel displacement, $w_{\max }$, bending moment, $M_{\max }$, and shear force, $Q_{\max }$, may be expressed, respectively, as:

$$
\begin{gathered}
w_{\max }=\frac{a_{\max } L^{2}}{V^{2}} f\left(\frac{k L^{4}}{E I}\right), \quad M_{\max }=\frac{a_{\max } E I}{V^{2}} g\left(\frac{k L^{4}}{E I}\right), \\
Q_{\max }=\frac{a_{\max } E I}{V^{2} L} h\left(\frac{k L^{4}}{E I}\right)
\end{gathered}
$$

assuming that the tunnel behaves as a Euler Bernoulli beam, and by

$$
\begin{gathered}
w_{\max }=\frac{a_{\max } L^{2}}{V^{2}} f^{*}\left(\frac{k L^{4}}{E I}, s\right), \quad M_{\max }=\frac{a_{\max } E I}{V^{2}} g^{*}\left(\frac{k L^{4}}{E I}, s\right), \\
Q_{\max }=\frac{a_{\max } E I}{V^{2} L} h^{*}\left(\frac{k L^{4}}{E I}, s\right)
\end{gathered}
$$

when the tunnel behaves as a Timoshenko beam, with $s$ being a parameter defined as

$s=\frac{E I}{G A_{\mathrm{c}} L^{2}}=\frac{2(1+v)}{k_{\mathrm{ef}}}\left(\frac{\kappa}{L}\right)^{2}$

where $v$ is the Poisson ratio of the tunnel material and $\kappa$ the radius of gyration of the tunnel cross section.

Figs. 13 show the functions $f$ and $f^{*}, g$ and $g^{*}$, and $h$ and $h^{*}$, respectively. After inspection of these latter figures, absolute values of the displacements, bending moments, and shear forces increase as the ratio $k L^{4} / E I$ becomes greater. Regardless of the type of the approach used for the tunnel behaviour or the ratio $s$, the values of the maximum tunnel displacement (Fig. 1) become equal for each value of the ratio $k L^{4} / E I$ greater than $8 \times 10^{3}$. For values of $k L^{4} / E I$ less than $8 \times 10^{3}$, Timoshenko's beam approach leads to higher maximum tunnel displacements than those predicted by the Euler Bernoulli beam theory, and the larger the $s$ ratio the greater the maximum tunnel displacement value.

For $k L^{4} / E I$ values of less than 40 , the results concerning maximum bending moment and shear force (Figs. 2 and 3 ) are independent of the beam theory used, whereas for values of such a ratio greater than 40 , Euler Bernoulli beam approach leads to higher values of the maximum bending moment and shear force. In the last range of possible values of that ratio and for the Timoshenko beam approach, the bending moment and the shear force become greater as the ratio $s$ decreases.

\section{Summary and conclusions}

A procedure to solve the seismic longitudinal analysis of a tunnel has been presented considering two different tunnel behaviours (Euler Bernoulli or Timoshenko beams). Seismic input consists of surface waves (Rayleigh distortional component and Love waves). The closed form solutions of the differential equations give the transverse tunnel displacement, the bending moment, and the shear force at any section of the tunnel. Dimensional analysis of the results was made to provide engineers with a design tool. Timoshenko's beam approach predicts maximal values of tunnel displacements, bending mo ments, and shear forces below those given by the Euler Bernoulli

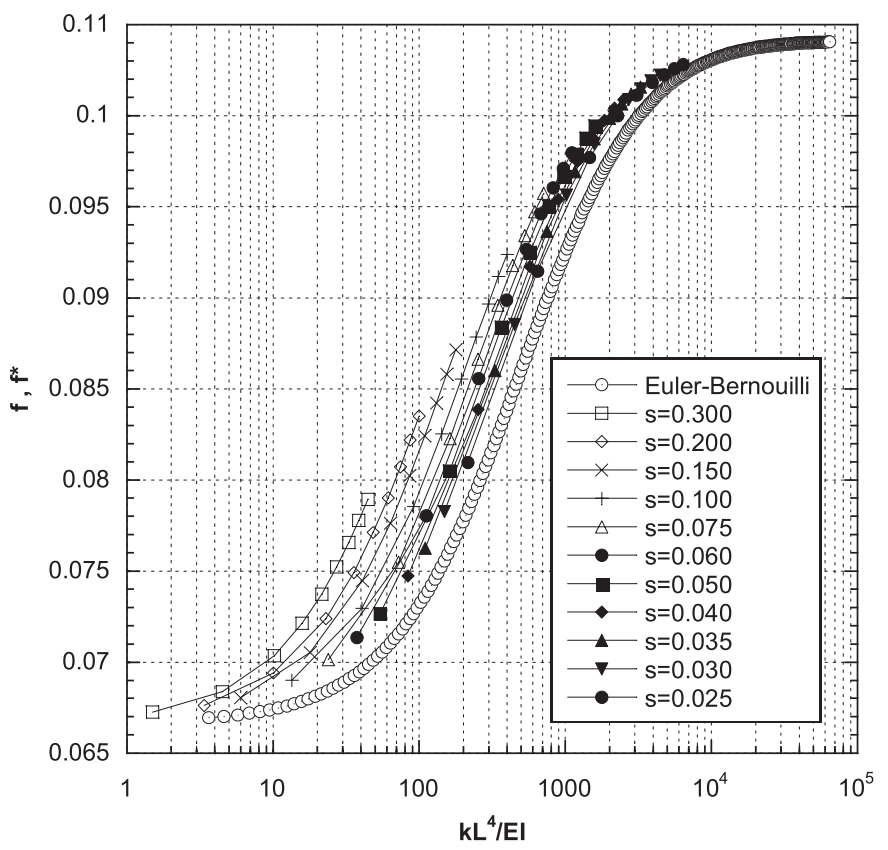

Fig. 1. Functions $f$ and $f^{*}$.

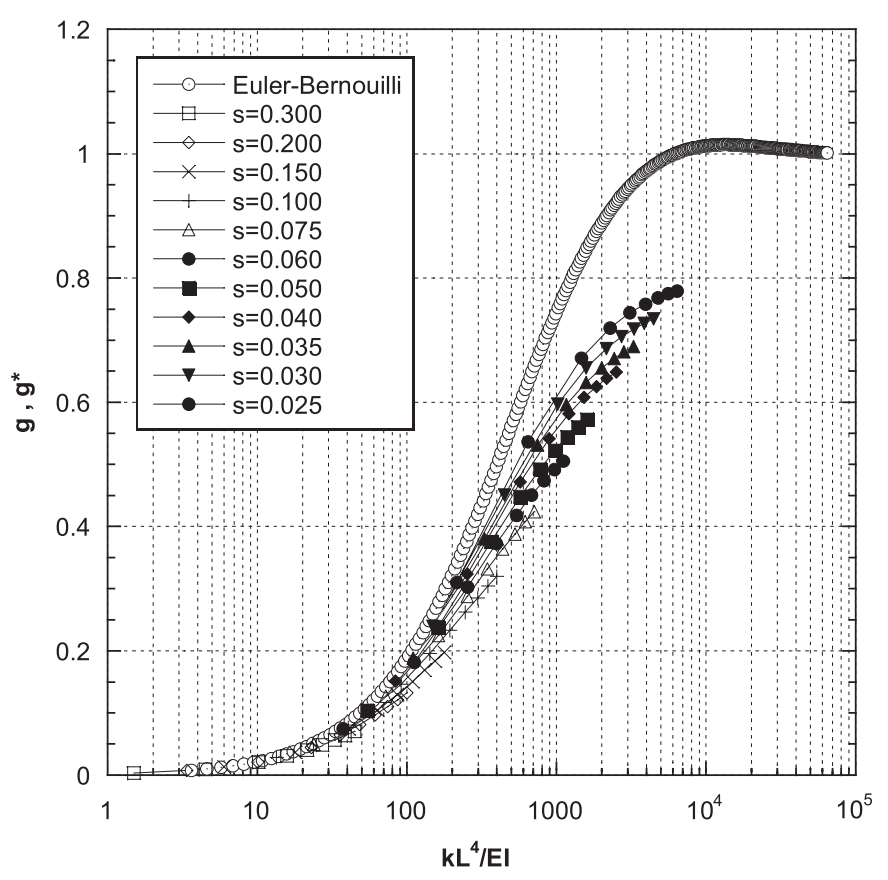

Fig. 2. Functions $g$ and $g^{*}$. 


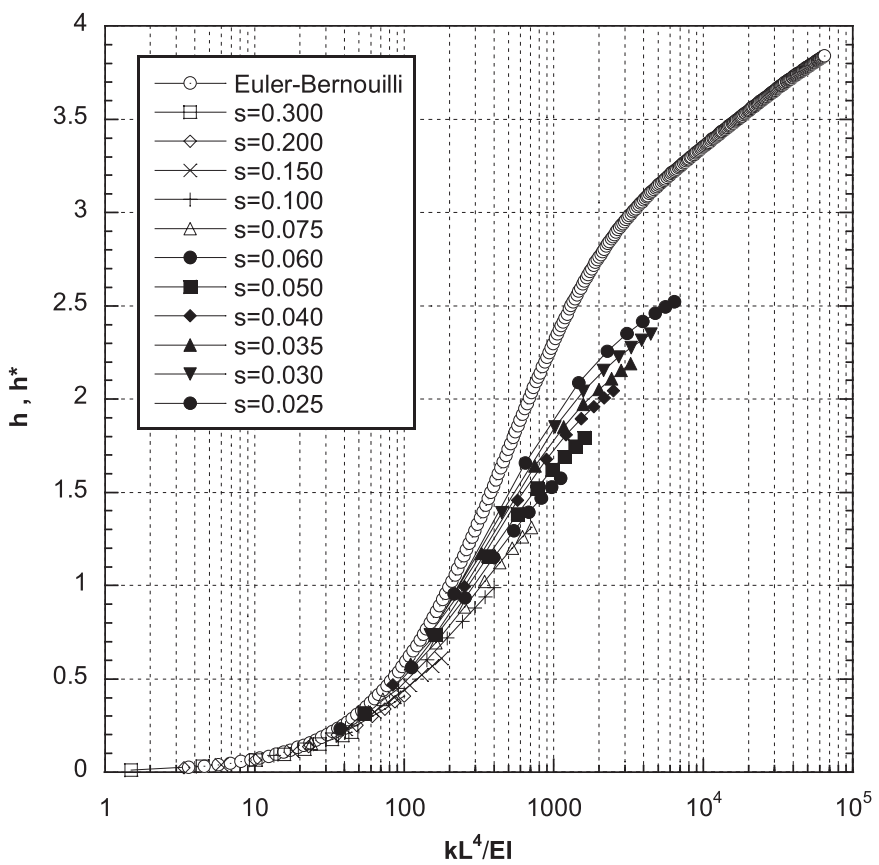

Fig. 3. Functions $h$ and $h^{*}$

theory. These latter magnitudes decrease as the ratio $k L^{4} / E I$ increases, although threshold values of this ratio have been found from which the values of the considered magnitudes predicted from these two beam theories do not significantly differ. In Timoshenko's beam approach, maximal bending moment and shear force in the tunnel become greater as the ratio $s$ decreases.

\section{Acknowledgement}

Authors are indebted to the Spanish Ministerio de Fomento (Project 80008/A04) for the financial support of this work.

\section{Appendix A. Parameters appearing in Eqs. (10) and (11)}

$$
\begin{aligned}
p= & \frac{a_{\max }}{v^{2}}\left[\begin{array}{ll}
\frac{8 E I}{A_{c} G L^{2}} & 1
\end{array}\right], \quad r=\frac{4 a_{\max }}{3 v^{2} L^{2}}, \\
C= & \frac{1}{4} f_{4}\left(\frac{p}{2}+\frac{3}{2} r\left(\frac{L}{2}\right)^{2}\right) \\
& +\frac{1}{4} f_{2} \frac{3}{2} r L, \quad D=\frac{1}{4} f_{3}\left(\frac{p}{2}+\frac{3}{2} r\left(\frac{L}{2}\right)^{2}\right) \quad \frac{1}{4} f_{1} \frac{3}{2} r L
\end{aligned}
$$

$f_{1}=\beta \sin \beta \frac{L}{2} \sinh \alpha \frac{L}{2}+\alpha \cos \beta \frac{L}{2} \cosh \alpha \frac{L}{2}$ $f_{2}=\beta \cos \beta \frac{L}{2} \cosh \alpha \frac{L}{2}+\alpha \sin \beta \frac{L}{2} \sinh \alpha \frac{L}{2}$

$f_{3}=\left(\begin{array}{ll}\alpha^{2} & \beta^{2}\end{array}\right) \cos \beta \frac{L}{2} \sinh \alpha \frac{L}{2} \quad 2 \alpha \beta \sin \beta \frac{L}{2} \cosh \alpha \frac{L}{2}$

$f_{4}=\left(\begin{array}{ll}\alpha^{2} & \beta^{2}\end{array}\right) \sin \beta \frac{L}{2} \cosh \alpha \frac{L}{2}+2 \alpha \beta \cos \beta \frac{L}{2} \sinh \alpha \frac{L}{2}$

$\Delta=f_{1} f_{4} \quad f_{2} f_{3}$

\section{Appendix B. . Parameters appearing in Eq. (14)}

$$
\begin{aligned}
& p=\frac{a_{\mathrm{max}}}{3 v^{2} L^{2}}, \quad q=\frac{a_{\mathrm{max}}}{2 v^{2}}, \quad r=\frac{a_{\max }}{v^{2}}\left[\frac{5 L^{2}}{48}+\frac{8 E I}{L^{2} k}\right], \\
& C_{\mathrm{w}}=\frac{1}{\alpha+\left(\beta^{2} / \alpha\right)}\left(N_{\mathrm{w}} \quad \frac{\beta}{a} M_{\mathrm{w}}\right), \quad D_{\mathrm{w}}=\frac{N_{\mathrm{w}}}{\beta} \quad \frac{\alpha}{\beta} C_{\mathrm{w}}
\end{aligned}
$$

in which $M_{\mathrm{w}}$ and $N_{\mathrm{w}}$ are

$M_{\mathrm{w}}=\frac{E I}{A_{\mathrm{c}} G}\left(2 C \alpha \beta+D\left(\alpha^{2} \quad \beta^{2}\right)\right) \quad D$

$N_{\mathrm{w}}=\frac{E I}{A_{\mathrm{c}} G}\left(C\left(\alpha^{2} \quad \beta^{2}\right)+2 D \alpha \beta\right) \quad C$

\section{References}

[1] Hashash YMA, Tseng WS, Krimotat A. Seismic soil-structure interaction analysis for immersed tube tunnels retrofit. Geotechnical Earthquake Engineering Soil Mechanics III 2, ASCE Geotechnical Special Publication no 75, 1998. p. 1380-91.

[2] Wang J. Seismic design of tunnels: a simple state-of-the-art design approach, Monograph 7. Parsons Brinckerhoff Quade \& Douglas, Inc.; June 1993.

[3] Benjamin Indrawan. Seismic design of tunnel structures. Jakarta: P.T., Pidelta Strukturindo; 2002

[4] Stamos AA, Beskos DE. 3-D seismic response analysis of long lined tunnels in half-space. Soil Dyn Earthquake Eng 1996;15(2):111-8.

[5] Luco JE, Barros FCP. Seismic Response of a Cylindrical Shell Embedded in a Layered Viscoelastic Half-space. I: Formulation. Earthquake Eng Struct Dyn 1994;23:553-67.

[6] Constantopoulos IV, Motherwel JT, Hall JR. Dynamic analysis of tunnels. In: Proceedings of the third international conference in geomechanics, Aachen, April 1979.

[7] Navarro C, Samartín A. Simplified longitudinal seismic analysis of buried tunnels. Software Eng Workstations 1988;4(1):3-10.

[8] St. John CM, Zahrah TF. Aseismic design of underground structures. Tunnelling Underground Space Technol 1987;2(2):165-97.

[9] Karadeniz H. Earthquake analysis of buried structures and pipelines base on Rayleigh wave propagation. Int J Offshore Polar Eng 2001:11(2):133-40.

[10] Hutchinson JR. Shear coefficients for Timoshenko beam theory. J Appl Mech 2001;68:87-92.

[11] Yin JH. Closed-form solution for reinforced Timoshenko beam on elastic foundation. J Eng Mech 2000:868-74. 